\title{
10008 A Novel Technique to Retrieve a Snagged Technical Stent Retriever: Technical Note Note
}

Norio Miyoshi, Kazutaka Shinomiya, Takanori Tanaka, and Ryunosuke Uranishi

Objective: Currently, there are no established approaches for removal of devices, such as stents, which sometimes become difficult to recover during endovascular treatment. We report a new method to successfully remove a stent that has become snagged during thrombus removal.

Case Presentation: An 82-year-old female who had undergone a mitral valve annuloplasty developed sudden aphasia, right hemiplegia, and right unilateral spatial neglect on postoperative day 10. Cranial MRI indicated occlusion of the horizontal segment of the left middle cerebral artery. During mechanical thrombectomy, a vasospasm snagged the stent, and re-sheathing attempts failed repeatedly. We wedged the microcatheter into the spasm site and slowly injected a solution containing $1 \mathrm{cc}$ of nicardipine, $2 \mathrm{cc}$ of contrast medium, and $2 \mathrm{cc}$ of heparin in normal saline intra-arterially. After several minutes, we retracted the Trevo wire slightly and easily removed the stent. The thrombus adhered to the retrieved stent. Post-retrieval imaging showed that the branch was completely recanalized.

Conclusion: In cases wherein a microwire or stent retriever becomes difficult to remove, we propose switching to a microcatheter with a sufficient diameter to allow vasodilator injection. If the microcatheter is difficult to remove, our method can be utilized by severing the hub, inserting a larger-bore catheter, and injecting vasodilators. Adding contrast medium to the intra-arterial injectate allows visualization of whether the solution has reached the spasm site. Furthermore, by injecting the solution through the wedged catheter, pooling of the solution at the spasm site can be confirmed.

Keywords $>$ snagged stent retriever, thrombectomy, vasodilator

\section{Introduction}

While rare, a microcatheter, ${ }^{1)}$ micro-guidewire, ${ }^{2)}$ or stent retriever ${ }^{3)}$ may be difficult to remove during endovascular treatment. As there is no established countermeasure, the following actions are commonly used: (1) temporary waitand-see, $\left.{ }^{4}\right)(2)$ removal via craniotomy, ${ }^{3)}$ or (3) leaving the device in place. ${ }^{1,2,4)} \mathrm{We}$ experienced a case of a retriever that became wedged and difficult to re-sheath due to a vasospasm in a patient with occlusion of the inferior branch (M2) of the middle cerebral artery (MCA). We successfully

Department of Neurosurgery, Bell Land General Hospital, Sakai, Osaka, Japan

Received: February 18, 2021; Accepted: May 23, 2021 Corresponding author: Norio Miyoshi. Department of Neurosurgery, Bell Land General Hospital, 500-3, Higashiyama, Naka-ku, Sakai, Osaka 599-8247, Japan Email: 09052450710@i.softbank.jp

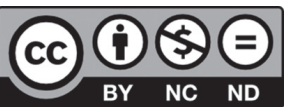

This work is licensed under a Creative Commons Attribution-NonCommercialNoDerivatives International License.

(C)2021 The Japanese Society for Neuroendovascular Therapy retrieved the stent by injecting a $\mathrm{Ca}^{2+}$-channel antagonist through the wedged catheter; this alleviated the vasospasm and allowed retrieval of the stent. No previous report has described a successful method for removing a stent retriever that became wedged due to vasospasm during endovascular treatment.

\section{Case Presentation}

The case describes an 82-year-old female who underwent mitral valve annuloplasty at the Department of Cardiovascular Surgery for mitral incompetency. Postoperative atrial fibrillation was observed, and a vitamin $\mathrm{K}$ antagonist was consequently administered as anticoagulant therapy. On day 9 after open heart surgery, cerebellar infarction occurred, but as the anticoagulant therapy was within the therapeutic range, patient progress was monitored. On postoperative day 10 , sudden aphasia, right hemiplegia, and right unilateral spatial neglect were observed (National Institute of Health Stroke Scale score [NIHSS] 13). Cranial MRI indicated occlusion of the horizontal segment of the left MCA (Fig. 1A). MRI before open heart surgery had revealed no 


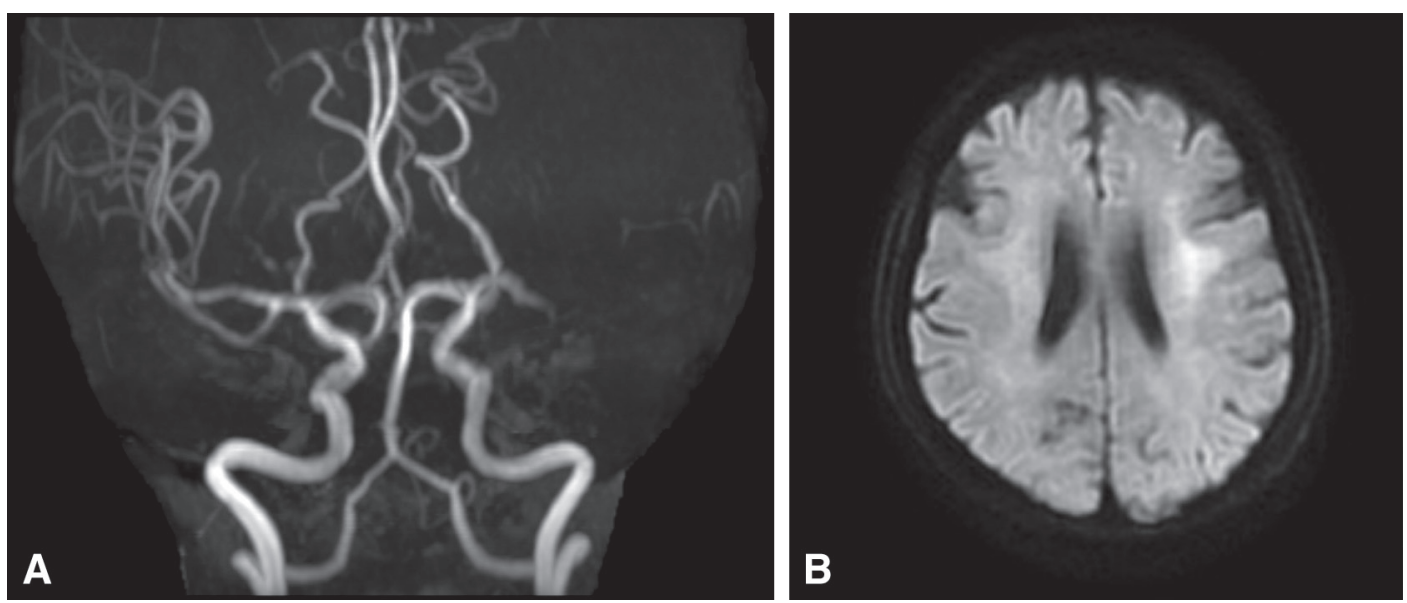

Fig. 1 (A) Preoperative MRIs showing occlusion of the horizontal segment of the left middle cerebral artery. (B) Preoperative diffusion-weighted imaging showing faint high-intensity signals in the deep white matter.

arteriosclerotic lesions at the site of the occlusion, suggesting that the occlusion was due to a cardiogenic embolism. Diffusion-weighted imaging indicated faint high-intensity signals in the deep white matter, with an Alberta Stroke Program Early CT score of 10 (Fig. 1B). On day 10 after open heart surgery, tissue plasminogen activator administration was impossible, and thus only mechanical thrombectomy could be performed.

A 9-Fr Optimo balloon guide catheter (Tokai Medical Products, Aichi, Japan) was inserted into the right femoral artery; imaging confirmed that the M2 was occluded, and the thrombus had migrated distally (Fig. 2). Since neurological function was not improving, we decided to pursue thrombectomy. Using a Penumbra ACE68 (Medico's Hirata, Osaka, Japan) as an intermediate catheter, together with a Marksman microcatheter (Medtronic, Minneapolis, MN, USA) and CHIKAI14 guidewire (Asahi Intecc, Aichi, Japan), we crossed the M2 occlusion. We continued with the push and fluff method using a TrevoXP ProVue Retriever $3 \times 20 \mathrm{~mm}$ (Stryker, Kalamazoo, MI, USA). After waiting for a few minutes, we introduced the Penumbra into the M1 and attempted to pull the stent to remove the thrombus but encountered strong resistance. Therefore, we decided to temporarily re-sheath the stent temporarily, and attempted to advance the Marksman microcathether into position, but were once again met with resistance. As it was likely that the captured thrombus was hindering the re-sheathing of the stent, we abandoned this approach. We then removed the microcatheter and inserted a larger-bore Penumbra 3MAX reperfusion catheter using a Trevo wire. Since the wire is fixed to the stent, it was unnecessary to extend the wire. We attempted to re-sheath the stent using the 3MAX but found that this was not possible.

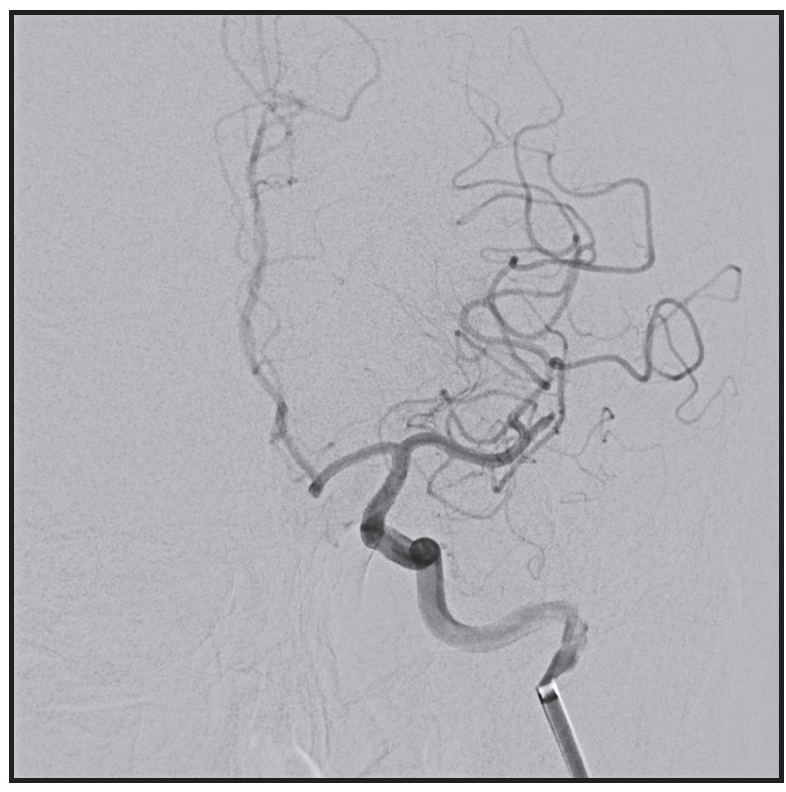

Fig. 2 Preoperative angiogram showing acute occlusion of the left inferior trunk of the middle cerebral artery.

To assess the situation accurately, we slowly injected contrast medium with the 3MAX wedged in the MCA inferior branch. Since the stenosis appeared at a site at which there was no arteriosclerotic lesion, we believed that vascular spasm had occurred, and the stent and captured thrombus were both snagged at this site (Fig. $\mathbf{3 A}$ and 3B). We therefore switched to a narrow-bore Rebar 18 catheter (Medtronic) to allow easy passage through the spasm site and once again attempted re-sheathing but were unsuccessful.

We then performed an intra-arterial injection of nicardipine through a Penumbra ACE68 that we inserted into the horizontal segment of the MCA to alleviate the vasospasm; however, we did not achieve any improvement. Imaging 

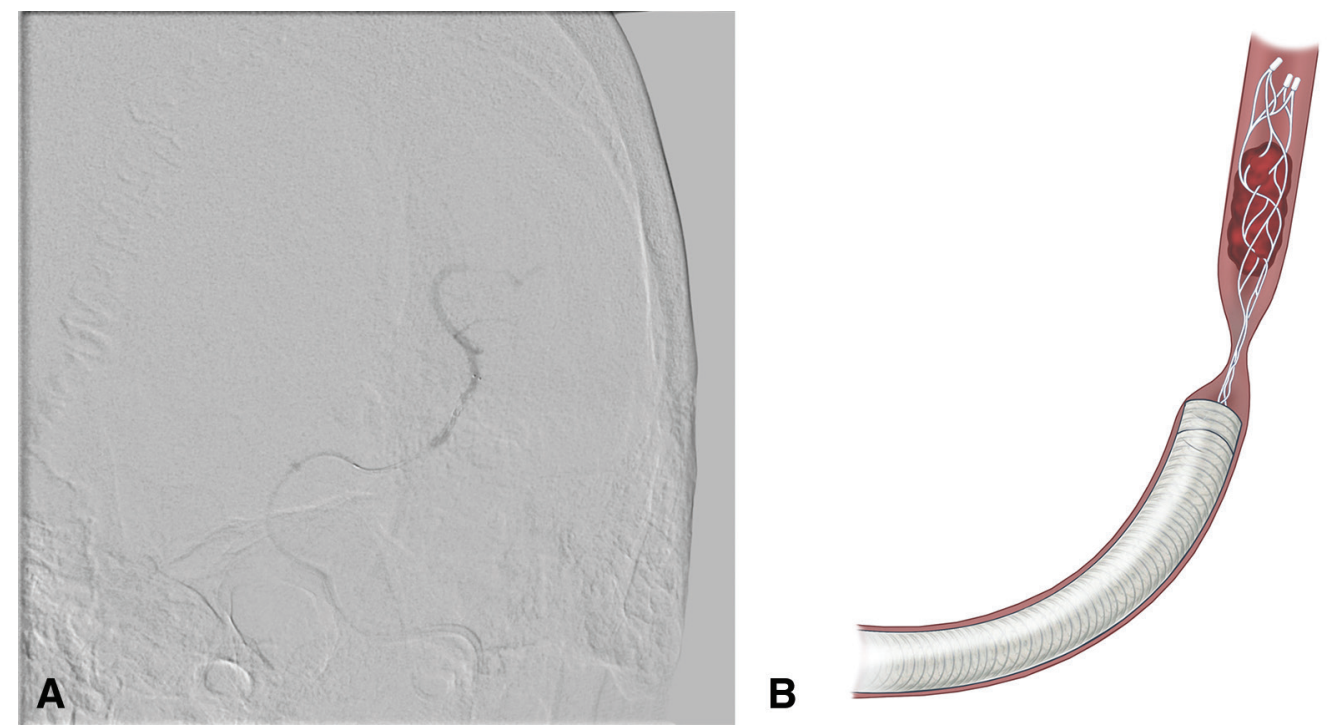

Fig. 3 (A) Intraoperative angiogram showing snagged stent retriever and spastic vessels, and wedged Penumbra 3MAX catheter. (B) Schematic image of (A) showing stent retriever and embolus being snagged by a spastic vessel.

through the Penumbra ACE68 indicated that the inferior branch contained no contrast medium, which suggested that the nicardipine did not reach the spasm site via intraarterial injection through this catheter.

Finally, we decided to wedge a microcatheter in the spasm site to perform another intra-arterial injection. Using the Trevo wire, we re-inserted the Penumbra 3MAX, wedged it into the vessel with the spasm, and slowly performed an intra-arterial injection of a solution containing $1 \mathrm{cc}$ of nicardipine, $2 \mathrm{cc}$ of contrast medium, and $2 \mathrm{cc}$ of heparin in normal saline using a $10 \mathrm{cc}$ syringe over $7 \mathrm{~min}$. Adding contrast medium to the solution allowed us to visualize whether the solution reached the spasm site, and by injecting the solution through the wedged catheter, we could confirm that the solution had pooled in the spasm site, even if the intra-arterial injection was interrupted (Fig. 4). After several minutes, we retracted the Trevo wire slightly and could retrieve the stent easily. The thrombus adhered to the retrieved stent, and images obtained immediately following retrieval showed that the branch was completely recanalized (Thrombolysis in Cerebral Infarction scale score 3).

Postoperative CT indicated a substantial amount of subarachnoid hemorrhage, but as it did not increase, patient progress was monitored (Fig. 5). On the day after surgery, neurological findings showed marked improvement, and the only remaining symptom was slight loss of coordination of the left upper and lower extremities (NIHSS 1). Postoperative MRI indicated a small high-intensity signal in the MCA region, while the high-intensity signal in the deep white matter had improved (Fig. 6). We observed no worsening of the vasospasm on MRA.

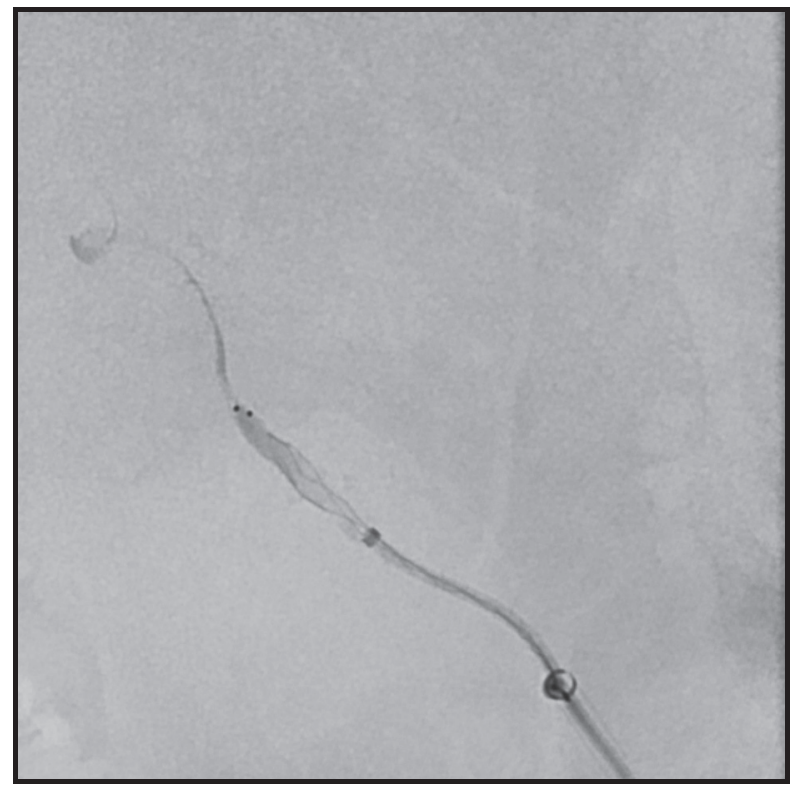

Fig. 4 Right anterior oblique angiogram showing wedge injection of a mixture of a $\mathrm{Ca}^{2+}$-channel blocker and contrast agent.

Twenty days later, after rehabilitation for slight lack of coordination, the patient was transferred to a step-down facility. After approximately 20 days of rehabilitation, there were no sequelae, and the patient was discharged home.

\section{Discussion}

While rare, difficulty in retrieving devices sometimes occurs during cerebrovascular treatment. The causes of such difficulty include entanglement with a previously inserted stent ${ }^{4)}$ and snagging of a device in a vasospasm. ${ }^{4}$ ) 


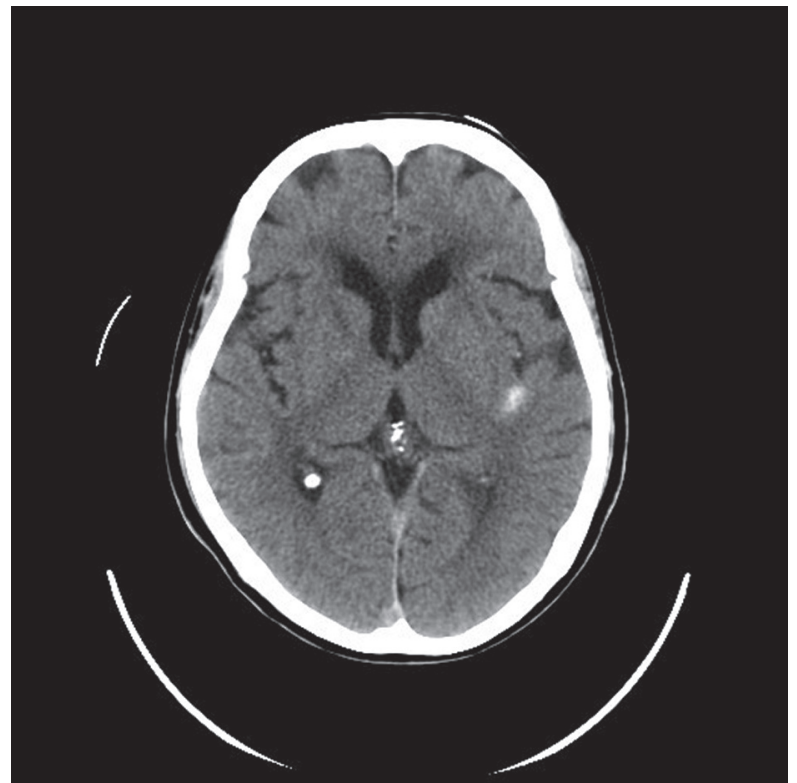

Fig. 5 Postoperative CT images showing a small subarachnoid hemorrhage in the left sylvian fissure.

A temporary wait-and-see approach is sometimes used when a device becomes lodged due to a vasospasm, while in other cases, a device may simply be left in place. However, this solution is linked to future complications. For instance, Konstas et al. reported a case in which a wire left in place caused aortic dissection. ${ }^{2}$ Koo et al. reported two cases in which the wire of a device that could not be retrieved broke and migrated within the chest cavity. ${ }^{4)}$ They discussed the possibility that the attachment of the device wire to the insertion tip of the catheter prevented the wire from moving freely, which may have led to the migration. Thus, they proposed that, when the device must be left in place, it should be done in such a way that ensures some freedom of movement. They also emphasized the importance of carefully following up on patients within whom a device has been left unintentionally. Craniotomy is also used to remove some devices. Yokoya et al. reported a case in which a stent retriever was removed via craniotomy. ${ }^{3)}$ Under microscopic observation, they noted that pulling on the stent retriever during craniotomy resulted in strong vascular deviation, which raised concern about damaging the perforating branch. Thus, they recommended that extreme caution should be exercised to prevent applying excessive stress on devices that cannot easily be retrieved.

In our case, we wedged a microcatheter (Penumbra $3 \mathrm{MAX}$ ) that was oversized relative to the diameter of the wire into the spasm site, injected vasodilator to relieve the vasospasm and were eventually successful in retrieving the device. To the best of our knowledge, this approach has not

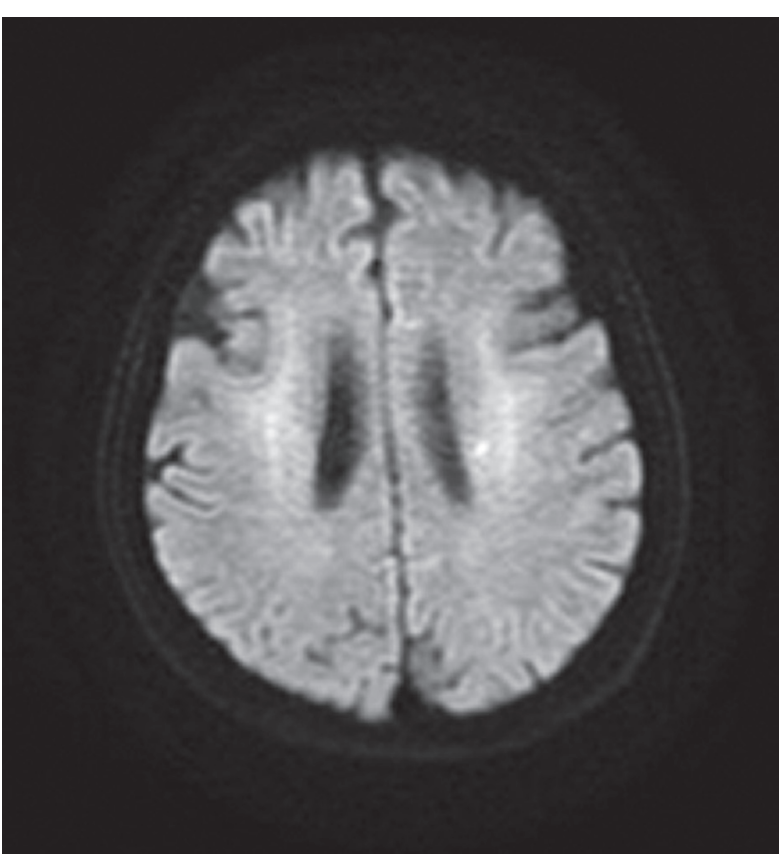

Fig. 6 Postoperative diffusion-weighted imaging showing a spot of high-intensity signal in the middle cerebral artery region, while the high-intensity signal in the deep white matter had improved.

been reported previously. Although we only report one case, we believe that this method is extremely useful and safe.

The merits of mixing contrast medium with the vasodilator are as follows: (1) it makes it possible to visualize whether the vasodilator has reached the spasm site; (2) when performed in combination with injection through a wedged catheter, it allows pooling of the vasodilator at the spasm site; (3) it allows determination of the treatment efficacy in real time; and (4) it allows early identification of extravasation. When we performed this approach through the intermediate catheter, it was difficult to reach the spasm site after vasodilator administration, so in our second attempt, we wedged the catheter prior to the injection, which allowed effective delivery of the vasodilator to the spasm site.

We believe that attempting our method has value in cases in which a device is difficult to retrieve due to vasospasm, regardless of the type of device. In cases in which a microwire or stent retriever becomes difficult to retrieve, we propose switching to a microcatheter with a sufficient diameter to allow injection of the vasodilator. In cases in which a microcatheter has become difficult to retrieve, our method can be utilized by severing the hub, inserting a larger-bore Penumbra, and injecting vasodilator.

A potential limitation of this method is the possibility of stent snagging due to arteriosclerosis. However, in many 
cases, it is difficult to distinguish between arteriosclerotic lesions and vascular spasms, and there is no harm in attempting to apply this method for arteriosclerotic lesion.

\section{Conclusion}

When devices that have been placed as part of endovascular treatment are impossible to retrieve, it can result in a critical situation that requires accurate countermeasures to avoid complications. In such a situation, the cause should be identified first, and if vasospasm is suspected, the following actions should be considered in order: (1) wait-and-see, for a short time; (2) insert an oversized catheter and inject vasodilator, and (3) consider craniotomy. When retrieval remains difficult in any of the above cases, the device by necessity must be left in place; however, in such cases, careful followup is required.

\section{Acknowledgments}

We would like to thank Editage (www.editage.com) for English language editing and LAIMAN (www.laiman. co.jp) for the schematic image.

\section{Disclosure Statement}

The authors declare that they have no conflicts of interest. This research did not receive any specific grant from funding agencies in public, commercial, or not-for-profit sectors.

\section{References}

1) Zoarski GH, Lilly MP, Sperling JS, et al. Surgically confirmed incorporation of a chronically retained neurointerventional microcatheter in the carotid artery. AJNR Am J Neuroradiol 1999; 20: 177-178.

2) Konstas AA, Pile-Spellman J. Aortic dissection associated with a neurointerventional guidewire retained in a perforating branch of the right posterior cerebral artery. Br J Radiol 2007; 80: e290-292.

3) Yokoya S, Hino A, Takezawa H, et al. Microsurgical removal of snagged stent retriever during endovascular embolectomy for acute ischemic stroke. World Neurosurg 2018; 111: 115-118.

4) Koo HW, Park W, Yang K, et al. Fracture and migration of a retained wire into the thoracic cavity after endovascular neurointervention: report of 2 cases. J Neurosurg 2017; 126: $354-359$. 